\title{
Ego depletion in visual perception: Ego-depleted viewers experience less ambiguous figure reversal
}

\author{
Marina C. Wimmer $^{1}$ - Steven Stirk ${ }^{1}$ Peter J. B. Hancock ${ }^{2}$
}

Published online: 22 February 2017

(C) Psychonomic Society, Inc. 2017

\begin{abstract}
This study examined the effects of ego depletion on ambiguous figure perception. Adults $(N=315)$ received an ego depletion task and were subsequently tested on their inhibitory control abilities that were indexed by the Stroop task (Experiment 1) and their ability to perceive both interpretations of ambiguous figures that was indexed by reversal (Experiment 2). Ego depletion had a very small effect on reducing inhibitory control (Cohen's $d=.15$ ) (Experiment 1). Ego-depleted participants had a tendency to take longer to respond in Stroop trials. In Experiment 2, ego depletion had small to medium effects on the experience of reversal. Ego-depleted viewers tended to take longer to reverse ambiguous figures (duration to first reversal) when naïve of the ambiguity and experienced less reversal both when naïve and informed of the ambiguity. Together, findings suggest that ego depletion has small effects on inhibitory control and small to medium effects on bottom-up and top-down perceptual processes. The depletion of cognitive resources can reduce our visual perceptual experience.
\end{abstract}

Keywords Ambiguous figures $\cdot$ Reversal $\cdot$ Bottom-up processes $\cdot$ Top-down processes $\cdot$ Ego depletion

Research over the past 100 years has used ambiguous figures, pictures with more than one interpretation, to examine the interplay of bottom-up and top-down processing in visual

Marina C. Wimmer

marina.wimmer@plymouth.ac.uk

1 School of Psychology, Cognition Institute, University of Plymouth, Plymouth, UK PL4 8AA

2 Psychology, Faculty of Natural Sciences, University of Stirling, Stirling, UK FK9 4LA perception (Long \& Batterman, 2012; Melcher \& Wade, 2006; Ward \& Scholl, 2015; Wimmer \& Doherty, 2011). Ambiguous figures evoke different interpretations while the physical properties of the stimulus itself remain unchanged. This switching between interpretations is termed "ambiguous figure reversal" (Long \& Toppino, 2004). Whilst a plethora of research has demonstrated the interplay of processes allowing reversal, in the current research we addressed how the "depletion" of cognitive resources reduces our visual perceptual experience.

Evidence from different fields has demonstrated that inhibitory processes play a key role in the reversal experience. Developmental research has shown that inhibitory ability allows 4- to 5-year-old children to experience reversal per se when informed of the ambiguity (Wimmer \& Doherty, 2011). Bilingual children who have superior inhibitory control (Carlson \& Meltzoff, 2008) are more likely to reverse ambiguous figures than their monolingual peers (Bialystok \& Shapero, 2005; Wimmer \& Marx, 2014). In addition to allowing reversal, inhibitory control can also reduce reversal. Adults can voluntarily control the perception of one interpretation when instructed to hold their first interpretation, demonstrating the role of top-down processes in visual perception (Hochberg \& Peterson, 1987; Mathes, Strüber, Stadler, \& Basar-Eroglu, 2006; Meng \& Tong, 2004; Peterson \& Gibson, 1991; Slotnik \& Yantis, 2005; Suzuki \& Peterson, 2000; Van Ee, van Dam, \& Brouwer, 2005). However, viewers cannot fully control their reversal rate as the instruction to hold one interpretation only leads to a decrease in reversal of between one half and one third over a 3-minute period (Strüber \& Stadler, 1999), highlighting the additional role of bottom-up processes in reversal.

The current research investigates the specific role of inhibitory processes in reversal and takes an opposite approach. If inhibitory control allows reversal per se, will the depletion of inhibitory 
processes directly interfere with reversal, thus, reduce it? To investigate the effect of inhibitory depletion on reversal, we use an ego-depletion method from social psychology. Ego depletion refers to "a temporary reduction in the self's capacity or willingness to engage in volitional action, (including controlling the environment, controlling the self, making choices, and initiating action) caused by prior exercise of volition" (Baumeister, Bratslavsky, Muraven, \& Tice, 1998, p. 1253). The underlying principle of ego depletion is that performance on a task requiring self-control will subsequently lead to a decrease in performance in an unrelated self-control task (Alós-Ferrer, Hügelschäfer, \& Li, 2015; Baumeister et al., 1998; Hagger, Wood, Stiff, \& Chatzisarantis, 2010). Specifically, participants who cross off some instances of the letter $e$ following complex rules watch a boring movie longer when it requires active quitting (pressing a button) than passive quitting (removing the hand from a button; Baumeister et al., 1998). Thus, participants' self-control is impaired after monitoring behaviour and overriding a habitual response, such as crossing off all instances of the letter $e$ (Baumeister et al., 1998).

The ego-depletion effect is demonstrated widely across a range of tasks, but the underlying mechanisms are not well understood. Ego depletion may reflect a low-level bottom-up process (Baumeister et al., 1998) or a high-level top-down process (Inzlicht, Schmeichel, \& Macrae, 2014). The energy model (Baumeister et al., 1998) purports that self-control is a limited resource. In a self-control task, energy is consumed and subsequent task performance requiring self-control will be reduced due to limited energy and its conservation, analogous to a tired muscle (Baumeister, 2014). For example, selfcontrol is linked to blood glucose levels and consuming a glucose drink reduces the ego depletion effect (Galliot et al., 2007). In contrast, self-control requirements may subsequently lead to reduced attention to cues that require control and reduced motivation to exert control (Inzlicht et al., 2014). For example, the ego-depletion effect is reduced when participants are motivated to perform a task such as thinking that their participation helps in finding Alzheimer's disease treatments (Muraven \& Slessareva 2003). The energy model can account for these findings too; motivation reduces the ego depletion effect because ego depleted participants can still exert conserved self-control if motivated beforehand (Baumeister, 2014). Overall, it is unclear what processes underlie ego depletion per se. However, given that ego depletion affects bottom-up processes (Baumeister, 2014) and top-down processes (Inzlicht et al., 2014), it should reduce ambiguous figure reversal involving both processes.

To test whether the depletion of inhibitory control leads to reduced reversal, we adapted the well-established ego depletion task (Baumeister et al., 1998) and measured the effect on inhibitory control, indexed by the Stroop task (Stroop, 1935) (Experiment 1) and reversal (Experiment 2). Specifically, it was examined whether ego depletion affects reversal when being naïve of the ambiguity (bottom up) versus being informed of both interpretations (top down). If ego depletion reflects a low-level process (Baumeister et al., 1998), then this should lead to reduced reversal under naïve conditions; whereas if it reflects a high-level process (Inzlicht et al., 2014), then this should reduce reversal under informed conditions.

\section{Experiment 1: Establishing ego-depletion effects on inhibitory control}

Here, the effects of ego depletion on inhibitory control were examined. Participants were either ego depleted using a wellestablished ego depletion task (Baumeister et al., 1998) or not depleted and then subsequently given the Stroop task, indexing inhibitory control.

\section{Method}

\section{Participants}

Overall, 214 adults ( 165 females, $M=22$ years, $S D=7$ years) recruited via the Plymouth University online participation system participated. They either received course credit or financial reimbursement.

\section{Design}

Half of the participants received an ego depletion task $(N=$ $113)$, and the other half an analogous control task $(N=101)$. After that, all participants received a computerized Stroop task. The experiment lasted around 30 minutes.

\section{Materials and procedure}

Participants in the control condition received a typewritten sheet of paper containing technical text (a page from a neuroscience article) and were instructed to cross off all instances of the letter $e$. Participants in the ego depletion condition were additionally told to only cross off an $e$ if it is "not adjacent to another vowel and more than one letter away from another vowel" (thus, one would not cross off the $e$ in pear or vowel) (Baumeister et al., 1998). Eight example words were provided, clarifying the instructions.

After that, all participants received a computerized version of the Stroop task on a standard PC $(1920 \times 1080$ resolution $)$, containing 100 word-reading, 100 colour-naming, and 100 interference trials. Each trial type was preceded by 10 practise trials. Trial type order was counterbalanced between participants. Stimuli comprised colour words (red, green, blue, yellow) written in red, green, blue, yellow, or black font. Colour words were aligned in a $2 \times 2$ square configuration in the centre of the screen, centred by a fixation cross where the mouse was positioned at the 
start of each trial. The target stimulus appeared below the square configuration and was displayed until participants gave a response via mouse click, followed by the next trial 1,000 ms apart. In word-reading trials, the target was a colour word in black font, and participants clicked on the according colour patch (e.g., "If the word BLUE appears, click on the blue colour patch"). In colour-naming trials, the target was a colour patch, and participants clicked on the according word (e.g., "If you see a BLUE patch, click on the word blue"). In interference trials, the target was a colour word, and participants clicked on the colour the word is written in (e.g., "If you see the word BLUE written in red, click on the word red). In interference trials, the colour word was incongruent with the colour the word was written in, in most trials (76 trials), mixed with 24 congruent trials to increase the interference effect.

\section{Results and discussion}

Accuracy on percentage of Stroop trials was at ceiling (ego depleted: $M=98.34$; control: $M=97.89$ ), and therefore no statistical analysis was conducted. Eight participants (three ego depleted and five controls) failed all interference trials and were excluded from analyses. The final analyses include participants who followed the ego-depletion instructions, that is, both rules $(N=82)$ and control instructions $(N=95)$.

We analysed mean response time in the Stroop task using bootstrap resampling to obtain effect sizes. There was barely a response time difference on overall Stroop response time between ego-depleted participants $(M=1,001 \mathrm{~ms})$ and controls ( $M=985 \mathrm{~ms})$, Cohen's $d=.095$.

However, it was of theoretical interest whether ego depletion would reduce inhibitory control rather than all-across-the-board response time. To isolate the inhibitory component, for each participant, the average of their response time in the two control conditions (word reading and colour naming) was subtracted from their mean response time in interference trials. In this inhibitory mean response time measure, ego-depleted participants $(M$ $=233 \mathrm{~ms}$ ) had a tendency to take longer to respond than control participants ( $M=207 \mathrm{~ms}$ ) Cohen's $d=0.15$. The same findings were obtained when response time in colour naming was used as comparison against the interference trials (interference latency colour naming latency); ego depleted $(M=234 \mathrm{~ms}$ ) versus controls $(M=205 \mathrm{~ms})$, Cohen's $d=.18$.

Thus, there is a small effect of ego depletion reducing inhibitory control. These findings do not support previous research revealing large ego depletion effects on inhibitory control (Johns et al., 2008). Can this small effect be a result of procedural differences of our Stroop version such as responding via mouse click or implementing the traditional blocked trial version as opposed to the more recently used item-by-item version? This seems unlikely as, if anything, the blocked version reveals larger inhibitory control effects than the item-by-item version (Ludwig, Borella, Tettamanti,
\& de Ribaupierre, 2010; Salo, Henik, \& Robertson, 2001). Thus, we would have expected to find at least equally large ego depletion effects on Stroop performance in a blocked version as in Johns et al.'s (2008) item-by-item version. Moreover, the Stroop effect is demonstrated widely across different response modalities (e.g., oral vs. pressing a keyboard button vs. typing in the word). At which stage of the task the Stroop effect emerges is subject to debate (during encoding or response selection), but evidence rules out that the Stroop effect occurs at response execution (Damian \& Freeman 2008; Logan \& Zbrodoff, 1998). Therefore, it is unlikely that procedural differences in the Stroop version can explain the differences in the magnitude of the ego depletion effect in our study $(d=.15)$ and in Johns et al. (2008) $(d=$ $.76)$.

\section{Experiment 2: The effects of ego depletion on ambiguous figure reversal}

Having established a small reduction of inhibitory control with the current ego depletion task, it was examined whether ego depletion reduces reversal (duration to first reversal and number of reversals) when naïve versus informed of ambiguity.

\section{Method}

\section{Participants}

Overall, 101 adults (69 females) ( $M=24$ years, $S D=11$ years) recruited via the Plymouth University online participation system took part and either received course credit or financial reimbursement.

\section{Design}

First, participants received the same ego depletion $(N=51)$ or control task $(N=50)$ as in Experiment 1 , followed by the ambiguous figure (AF) reversal task.

\section{Materials and procedure}

The ego depletion task was the same as in Experiment 1. The AF reversal task was computerized and ran on a standard PC $(1920 \times 1080$ resolution $)$ in Visual Basic.

Ambiguous figure (AF) reversal task The ambiguous duck/ rabbit $(11 \times 7.5 \mathrm{~cm}$; Jastrow, 1900$)$, man/mouse $(8.5 \times 7.5 \mathrm{~cm}$, Bugelski \& Alampay, 1961), mother/face $(9 \times 10.5 \mathrm{~cm}$; Fisher, 1967), and cowboy/Indian $(10 \times 11.5 \mathrm{~cm}$; Botwinick, 1961) were used (see Fig. 1). Participants sat approximately 1 $\mathrm{m}$ from the screen. 


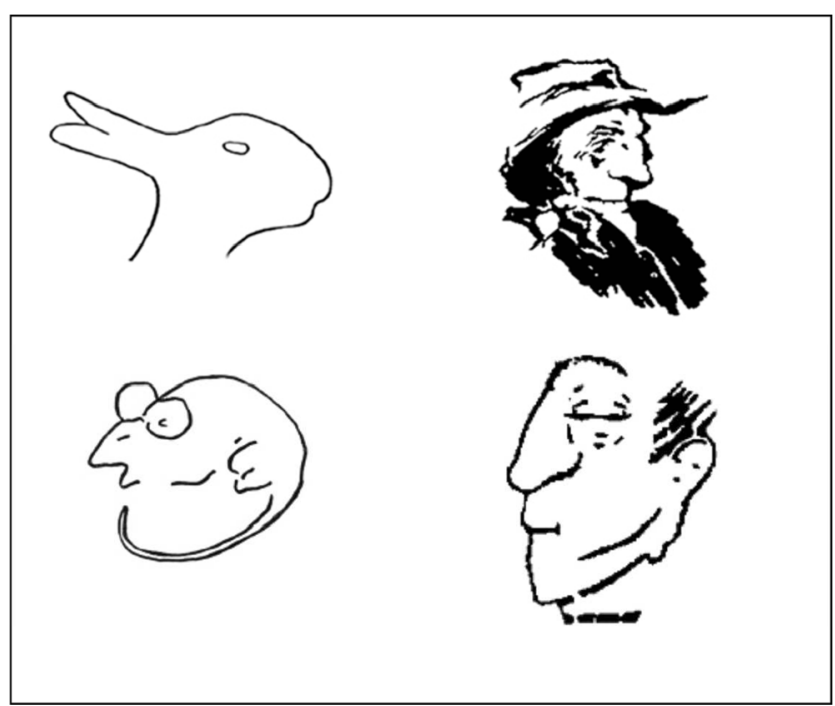

Fig. 1 AFs used in Experiment 2 (clockwise from top-left: duck/rabbit, cowboy/Indian, face/mother, man/mouse)

Before presenting the ambiguous figures, participants received two familiarization trials, each lasting 60 seconds. In one trial, the image changed physically (a horse morphed into a sheep), whereas in the other, no change occurred as the figure was unambiguous (a line drawing of a girl). Participants pressed the space bar whenever they thought that the image changed. The purpose of familiarization was to introduce the concept of change without the concept of ambiguity and to control for false positives (people reporting changes without perceiving them) and false negatives (people perceiving changes without reporting them). Two participants were identified as false positives, and their data were removed from further analyses.

After familiarization, in the naïve phase, participants were presented with the first ambiguous figure and uninformed of the ambiguity and alternative interpretations. Participants were asked what they saw (initial interpretation; e.g., "a duck"). Then, they indicated their perceptual changes via button press over 60 seconds. The program recorded the dependent variables, (i) when the first reversal occurred (duration to first reversal) and (ii) how often reversal occurred (reversal rate). This was repeated with the remaining three ambiguous figures (man/mouse, cowboy/Indian, face/mother). Ambiguous figures appeared in random order counterbalanced between participants. If participants reversed, then at the end of the naïve phase, they were asked what the alternative perceived interpretation was, ensuring perception of the alternative interpretation as indicated. Alternative labels were accepted as long as the participant was able to indicate relevant features of his or her stated changed interpretation (e.g., old woman instead of Indian or woman instead of mother with baby or man instead of face).

Then, the informed phase followed the same procedure, except that first, each figure was disambiguated by adding disambiguating context drawings for each interpretation and each interpretation was labelled (e.g., "This could be a duck, this could be a rabbit." "You will now be shown the ambiguous image for 60 seconds. Please press the space bar each time you see the image flip between pictures").

At the end, participants were asked whether they had seen any ambiguous figure before.

\section{Results and discussion}

Data of five outliers (2 standard deviations above the mean) in the number of reversals experienced in the naïve phase were removed from any further analysis.

Prior knowledge of ambiguous figures Depending on the type of ambiguous figure, there were considerable differences in whether it had been seen before, Friedman test, $\chi^{2}(3,94)=$ $73.9, p<.001$. Specifically, the duck/rabbit was more known (all $z \mathrm{~s}>5.06, p \mathrm{~s}<.001$ ) (by $45 \%$ of participants) than any other figure which did not differ (all $p \mathrm{~s}>.05$ ) (Wilcoxon Signed Ranks), except that the cowboy/Indian was more known than the man-mouse ( $p=.04$; face/woman: 7\%, cowboy/Indian: $11 \%$, man/mouse: $4 \%$ ). Thus, because of high familiarity with the duck/rabbit figure to compare naïve and informed phases, this figure was removed from any further analyses.

Ambiguous figure reversal We analysed mean time in seconds to first reversal (duration to first reversal) and mean number of reversals (reversal rate), using bootstrap resampling to obtain confidence intervals on both the mean values and the effect sizes. Figure 2 shows the means.

The effect of being informed about ambiguity is clear, with a marked decrease in time to first reversal and a large increase in the total number of reversals reported. There is a tendency for ego-depleted participants to take longer to report a reversal and see fewer in total. Figure 3 shows the effect size of the differences between groups in each condition.

The estimate of effect size for time to first reversal is 0.42 in the naïve condition, falling to 0.08 in the informed condition. For number of reversals, the effect size increases from 0.26 when naive to 0.35 when informed (the direction of the difference is opposite, as shown in Fig. 2; reversing the sign of Cohen's $d$ makes Fig. 3 clearer. We cannot confidently rule out an effect size of zero in any condition, due to large individual differences between participants. However, the pattern seems clear: while the tendency of ego-depleted participants to take longer to see a reversal disappears when they are informed of the ambiguity, they still make fewer reversals overall.

This pattern is consistent with reduced bottom-up processing in ego-depleted participants. After ego depletion, it takes longer to reverse when uninformed of ambiguity (bottom-up), 

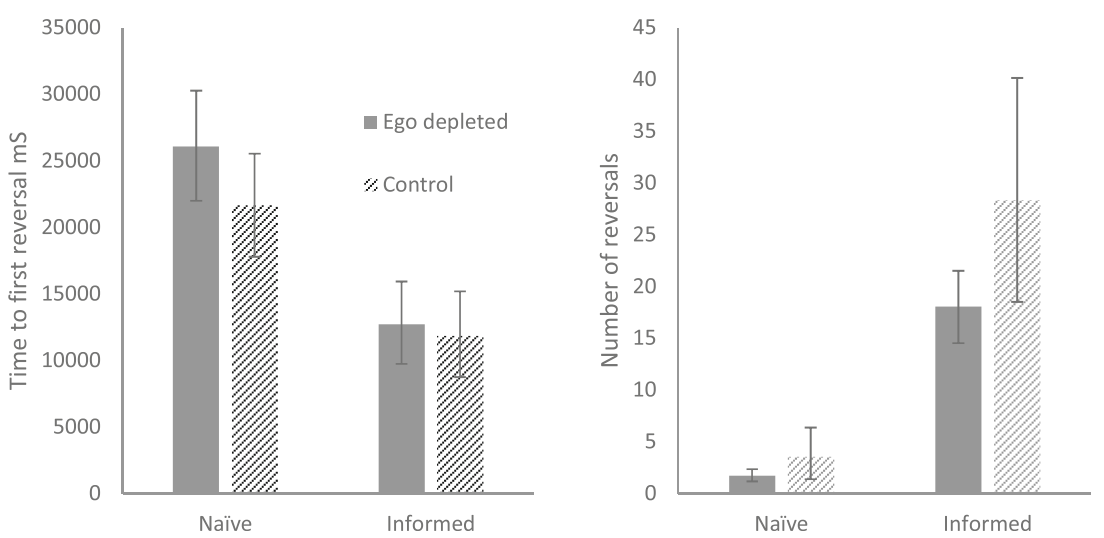

Fig. 2 Time to first reversal and number of reversals for ego-depleted and control participants, when naïve and informed about ambiguous figures. Error bars are $95 \% \mathrm{CI}$

which disappears when informed (top-down). The addition of this top-down information greatly increases the number of reversals seen, but has much the same effect on both control and ego-depleted participants, suggesting both are equally sensitive to top-down processing. Ego-depleted participants persist in seeing fewer reversal when informed, consistent with reduced bottom-up processing.

\section{General discussion}

The current aim was to examine whether the depletion of inhibitory control reduces the experience of reversal. Adapting an ego depletion paradigm from social psychology (Baumeister et al., 1998), inhibitory control was slightly reduced after ego depletion (Experiment 1), but it is important to stress that the effect is small. In Experiment 2, when egodepleted adults viewed ambiguous figures, they were less likely to reverse ambiguous figures. Thus, in addition to findings demonstrating that inhibitory control allows or stabilises reversal (e.g., Strüber \& Stadler, 1999; Wimmer \& Doherty, 2011), the current findings reveal that the depletion of inhibitory resources may reduce reversal. Reversal reduction occurred when both being naïve and informed of ambiguity, suggesting ego depletion effects on both bottom-up and topdown processes.

It is unclear what mechanisms underlie ego depletion, and there is debate whether ego depletion reflects the depletion of low-level processes (Baumeister et al., 1998) or reduces active motivation and attention to reach a goal or perform a task (Inzlicht et al., 2014). The current findings cannot directly answer this debate, and the reduction in reversal rate when informed of ambiguity can be a result of both neural fatigue effects (bottom-up) and lack of motivation to focus on the task (top-down). However, the additional finding that ego depletion increased response time to reverse initially when naïve about the ambiguity is in line with the suggestion that ego depletion particularly reduces bottom-up processing (Baumeister et al., 1998) rather than solely affecting topdown processing (Inzlicht et al., 2014). Thus, overall, a hybrid model of ego depletion involving both bottom-up and topdown processes seems more plausible.

However, the size of the ego depletion effect has recently been put into question in a preregistered replication study involving 23 laboratories (Hagger \& Chatzisarantis, 2016). Effect sizes on response time differences between egodepleted and control participants ranged between Cohen's $d$ $=-.06$ and .36 , with a $95 \%$ confidence interval. Moreover, task

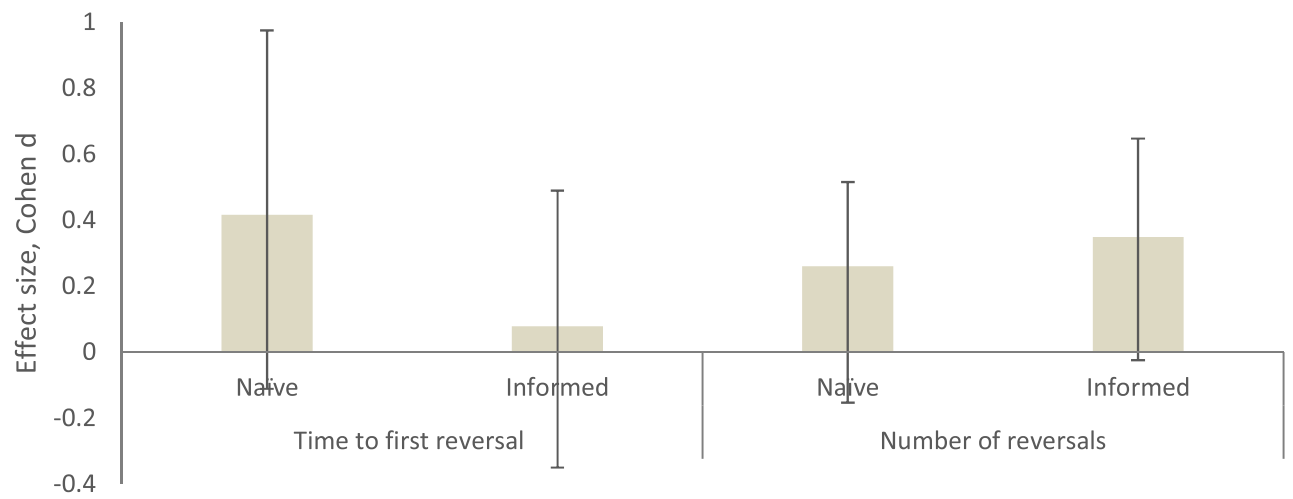

Fig. 3 Cohen's $d$ effect size for the difference between ego-depleted and control participants; sign-reversed for number of reversals. Error bars are 95\% CI 
performance varied greatly in accuracy both between egodepleted and control participants and across different laboratories (15\%-44\% of participants performing $<80 \%$ correct) (Hagger \& Chatzisarantis, 2016). Clearly, this raises the issue of the strength of the ego depletion effect and how minor procedural differences across laboratories, different populations, and variation in task performance affect the overall strength of the effect (Baumeister \& Vohs, 2016). Our single lab findings reveal a small ego depletion effect and do not support previous results showing strong effects of ego depletion on inhibitory Stroop performance (Johns, Inzlicht, \& Schmader, 2008). Power analysis (G*Power; Faul, Erdfelder, Lang, \& Buchner, 2007) suggests that we had sufficient power (174 participants needed) to detect large sized effects at $d=.5$. In Experiment 2, the ego depletion effect was larger, demonstrating novel effects in visual perception. Thus, the current research supports demonstrations of the effect across a wide domain of tasks (Hagger et al., 2010), but raises the question of the size of the effect.

Furthermore, findings from the ego depletion literature are at odds with the traditional cognitive literature on sequential modulation effects. When a task poses response conflict, then inhibitory control performance is enhanced in a second selfcontrol task due to activated cognitive control or priming (Botvinick, Braver, Barch, Carter, \& Cohen, 2001; Mayr, Awh, \& Laurey, 2003). Specifically, responding to an incongruent Flanker task trial reduces response time on an immediately followed incongruent number Stroop trial when participants are initially aware of trial difficulty (Fernandez-Duque \& Knight, 2008). Crucially, the sequential modulation effect occurs on a trial-by-trial basis when task type varies across trials as opposed to the sequential task paradigm in ego depletion. Thus, when the cognitive system is put under response conflict across different tasks, it can enhance cognitive control across tasks online on a trial-by-trial basis (cognitive literature on sequential modulation), but not when the task flow is interrupted (ego depletion literature). This difference between online task engagement and task interruption may cause participants to show opposite self-control effects. Future research might want to directly investigate this claim.

Overall, current findings provide novel insights into the functional dependence of inhibitory processes on the disambiguation of visual information under naïve and informed conditions. The depletion of inhibitory control may reduce our visual perceptual experience of reversal.

\section{References}

Alós-Ferrer, C., Hügelschäfer, S., \& Li, J. (2015). Self-control depletion and decision making. Journal of Neuroscience, Psychology, and Economics, 8, 203-216. doi:10.1037/npe0000047
Baumeister, R. F. (2014). Self-regulation, ego depletion, and inhibition. Neuropsychologia, 65, 313-319. doi:10.1016/j.neuropsychologia. 2014.08.012

Baumeister, R. F., Bratslavsky, E., Muraven, M., \& Tice, D. M. (1998). Ego depletion: Is the active self a limited resource? Journal of Personality and Social Psychology, 74, 1252-1265. doi:10.1037/ 0022-3514.74.5.1252

Bialystok, E., \& Shapero, D. (2005). Ambiguous benefits: The effect of bilingualism on reversing ambiguous figures. Developmental Science, 8, 595-604. doi:10.1111/j.14677687.2005.00451.x

Botvinick, M. M., Braver, T. S., Barch, D. M., Carter, C. S., \& Cohen, J. D. (2001). Conflict monitoring and cognitive control. Psychological Review, 108, 624-652. doi:10.1037/0033-295X.108.3.624

Botwinick, J. (1961). Husband and father-in-law: A reversible figure. American Journal of Psychology, 74, 312-113.

Bugelski, B. R., \& Alampay, D. A. (1961). The role of frequency in developing perceptual sets. Canadian Journal of Psychology, 15, 205-211. doi:10.1037/h0083443

Carlson, S. M., \& Meltzoff, A. M. (2008). Bilingual experience and executive functioning in young children. Developmental Science, 11, 282-298. doi:10.1111/j.1467-7687.2008.00675.x

Damian, M. F., \& Freeman, N. H. (2008). Flexible and inflexible response components: A Stroop study with typewritten output. Acta Psychologica, 128, 91-101. doi:10.1016/j.actpsy.2007.10.002

Faul, F., Erdfelder, E., Lang, A.-G., \& Buchner, A. (2007). G*Power 3: A flexible statistical power analysis program for the social, behavioral, and biomedical sciences. Behavior Research Methods, 39, 175-191. doi:10.3758/BF03193146

Fernandez-Duque, D., \& Knight, M. B. (2008). Cognitive control: Dynamic, sustained, and voluntary influences. Journal of Experimental Psychology: Human Perception and Performance, 34, 340-355. doi:10.1037/0096-1523.34.2.340

Fisher, G. (1967). Measuring ambiguity. American Journal of Psychology, 80, 541-557. doi:10.2307/1421187

Galliot, M. T., Baumeister, R. F., DeWall, C. N., Maner, J. K., Plant, E. A., Tice, D. M., \& Schmeichel, B. J. (2007). Self-control relies on glucose as a limited energy source: Willpower is more than a metaphor. Journal of Personality and Social Psychology, 92, 325-336. doi:10. 1037/0022-3514.92.2.325

Hagger, M. S., Wood, C., Stiff, C., \& Chatzisarantis, N. L. D. (2010). Ego depletion and the strength model of self-control: A meta-analysis. Psychological Bulletin, 136, 495-525. doi:10.1037/a0019486

Hochberg, J., \& Peterson, M. A. (1987). Piecemeal organization and cognitive components in object perception: Perceptually coupled responses to moving objects. Journal of Experimental Psychology: General, 116, 370-380. doi:10.1037/0096-3445.116.4.370

Inzlicht, M., Schmeichel, B. J., \& Mcrae, N. C. (2014). Why self-control seems (but may not be) limited. Trends in Cognitive Sciences, 18, 127-133. doi:10.1016/j.tics.2013.12.009

Jastrow, J. (1900). Fact and fable in psychology. Oxford: Houghton Mifflin.

Johns, M., Inzlicht, M., \& Schmader, T. (2008). Stereotype threat and executive resource depletion: Examining the influence of emotion regulation. Journal of Experimental Psychology: General, 137, 691-705. doi:10.1037/a0013834

Logan, G. D., \& Zbrodoff, J. N. (1998). Stroop-type interference: Congruity effects in color naming with typewritten responses. Journal of Experimental Psychology: Human Perception and Performance, 24, 978-992. doi:10.1037/0096-1523.24.3.978

Long, G. M., \& Batterman, J. M. (2012). Dissecting perceptual processes with a new tri-stable reversible figure. Perception, 41, 1163-1185. doi: $10.1068 / \mathrm{p} 7313$

Long, G. M., \& Toppino, T. C. (2004). Enduring interest in perceptual ambiguity: Alternating views of reversible figures. Psychological Bulletin, 130, 748-768. doi:10.1037/0033-2909.130.5.748 
Ludwig, C., Borella, E., Tettamanti, M., \& de Ribaupierre, A. (2010). Adult age differences in the color Stroop test: A comparison between an itemby-item and blocked version. Archives of Gerontology and Geriatrics, 51, 135-142. doi:10.1016/j.archger.2009.09.040

Mathes, B., Strüber, D., Stadler, M. A., \& Basar-Eroglu, C. (2006). Voluntary control of Necker cube reversals modulates the EEG delta- and gamma-band response. Neuroscience Letters, 402, 145149. doi:10.1016/j.neulet.2006.03.063

Mayr, U., Awh, E., \& Laurey, P. (2003). Conflict adaptation effects in the absence of executive control. Nature Neuroscience, 6, 450-452. doi: $10.1038 / \mathrm{nn} 1051$

Melcher, D., \& Wade, N. J. (2006). Cave art interpretation II. Perception, 35, 719-722. doi:10.1068/p3506ed

Meng, M., \& Tong, F. (2004). Can attention selectively bias bistable perception? Differences between binocular rivalry and ambiguous figures. Journal of Vision, 4, 539-551. doi:10.1167/4.7.2

Muraven, M., \& Slessareva, E. (2003). Mechanisms of self-control failure: Motivation and limited resources. Personality and Social Psychology Bulletin, 29, 894-906. doi:10.1177/0146167203029007008

Peterson, M. A., \& Gibson, B. S. (1991). Directing spatial attention within an object: Altering the functional equivalence of shape descriptions. Journal of Experimental Psychology: Human Perception and Performance, 17, 170-182. doi:10.1037/0096-1523.17.1.170

Salo, R., Henik, A., \& Robertson, L. C. (2001). Interpreting Stroop interference: An analysis of differences between task versions. Neuropsychology, 15, 462-471. doi:10.1037/08944105.15.4.462
Slotnik, S. D., \& Yantis, S. (2005). Common neural substrates for the control and effects of visual attention and perceptual bistability. Cognitive Brain Research, 24, 97-108. doi:10.1016/j.cogbrainres. 2004.12.008

Stroop, J. R. (1935). Studies of interference in serial verbal reactions. Journal of Experimental Psychology, 18, 643-662. doi:10.1037/ h0054651

Strüber, D., \& Stadler, M. (1999). Differences in top-down influences on the reversal rate of different categories of reversible figures. Perception, 28, 1185-1196. doi:10.1068/p2973

Suzuki, S., \& Peterson, M. A. (2000). Multiplicative effects of intention on the perception of bistable apparent motion. Psychological Science, 11, 202-209. doi:10.1111/1467-9280.00242

Van Ee, R., van Dam, L. C. J., \& Brouwer, G. J. (2005). Voluntary control and the dynamics of perceptual bi-stability. Vision Research, 45, 4155. doi:10.1016/j.visres.2004.07.030

Ward, E. J., \& Scholl, B. J. (2015). Inattentional blindness reflects limitations on perception, not memory: Evidence from repeated failures of awareness. Psychonomic Bulletin \& Review, 22, 722-727. doi:10. 3758/s13423-014-0745-8

Wimmer, M. C., \& Doherty, M. J. (2011). The development of ambiguous figure perception. Monographs of the Society for Research in Child Development, 76(1), 1-130.

Wimmer, M. C., \& Marx, C. (2014). Inhibitory processes in visual perception: A bilingual advantage. Journal of Experimental Child Psychology, 126, 412-419. 